Page 7, Figure 6, Table 0

-Supporting Information-

\title{
Alternating Current Influences Anaerobic Electroactive Biofilm
}

\section{Activity}

Xin Wang ${ }^{1}$, Lean Zhou, ${ }^{1}$, Lu Lu ${ }^{2}$, Fernanda Leite Lobo ${ }^{2}$, Nan $\mathrm{Li}^{3}$, Heming Wang ${ }^{2}$, Jaedo Park ${ }^{4}$ and Zhiyong Jason Ren ${ }^{2} *$

${ }^{1}$ MOE Key Laboratory of Pollution Processes and Environmental Criteria / Tianjin Engineering Center of Environmental Diagnosis and Contamination Remediation, Nankai University, No. 38 Tongyan Road, Jinnan District, Tianjin 300350, China

${ }^{2}$ Department of Civil, Environmental, and Architectural Engineering, University of Colorado Boulder, Boulder, CO 80309, USA

3 Tianjin Key Lab of Indoor Air Environmental Quality Control, School of Environmental Science and Engineering, Tianjin University, No. 92 Weijin Road, Nankai District, Tianjin 300072, China

${ }^{4}$ Department of Electrical Engineering, University of Colorado Denver, Denver, CO 80204, USA

*Corresponding Author: Phone: (303) 492-4137; E-mail: Zhiyong.Ren@ colorado.edu 


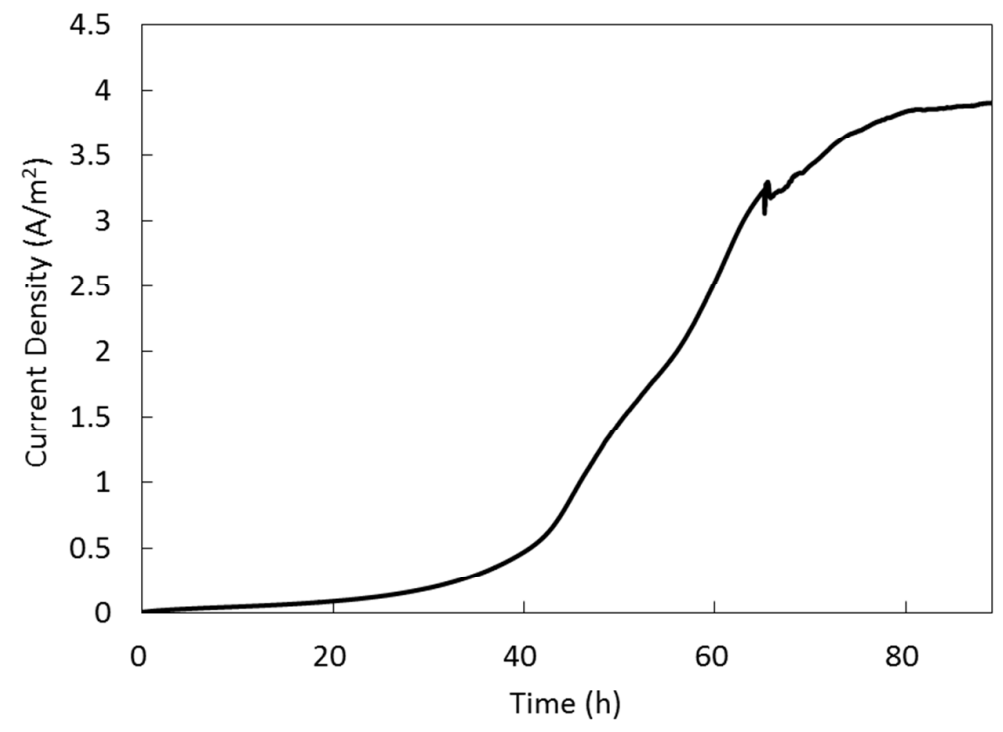

Figure S1 Typical current curve of BES during start-up period.

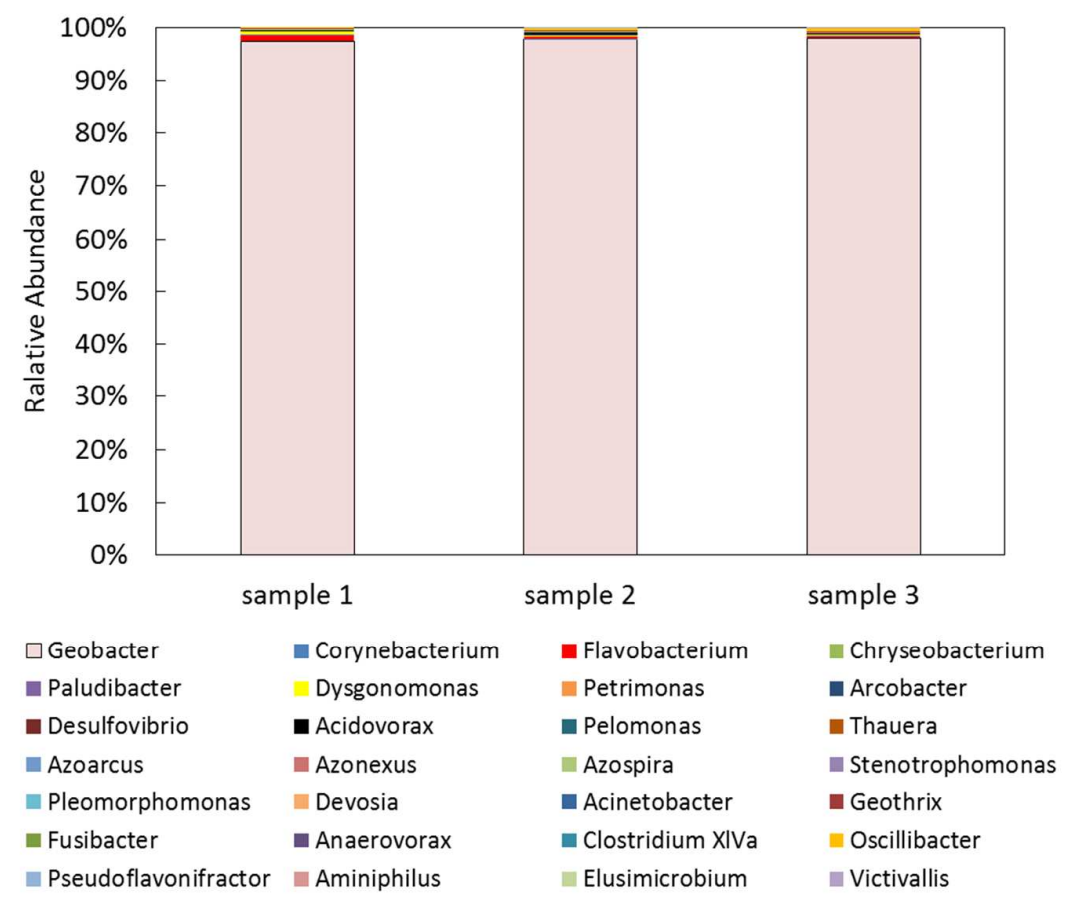

Figure S2 Taxonomic classification of bacterial communities at genus level of three random samples acclimated in different batches using microbial fuel cell effluent as the inoculum. 

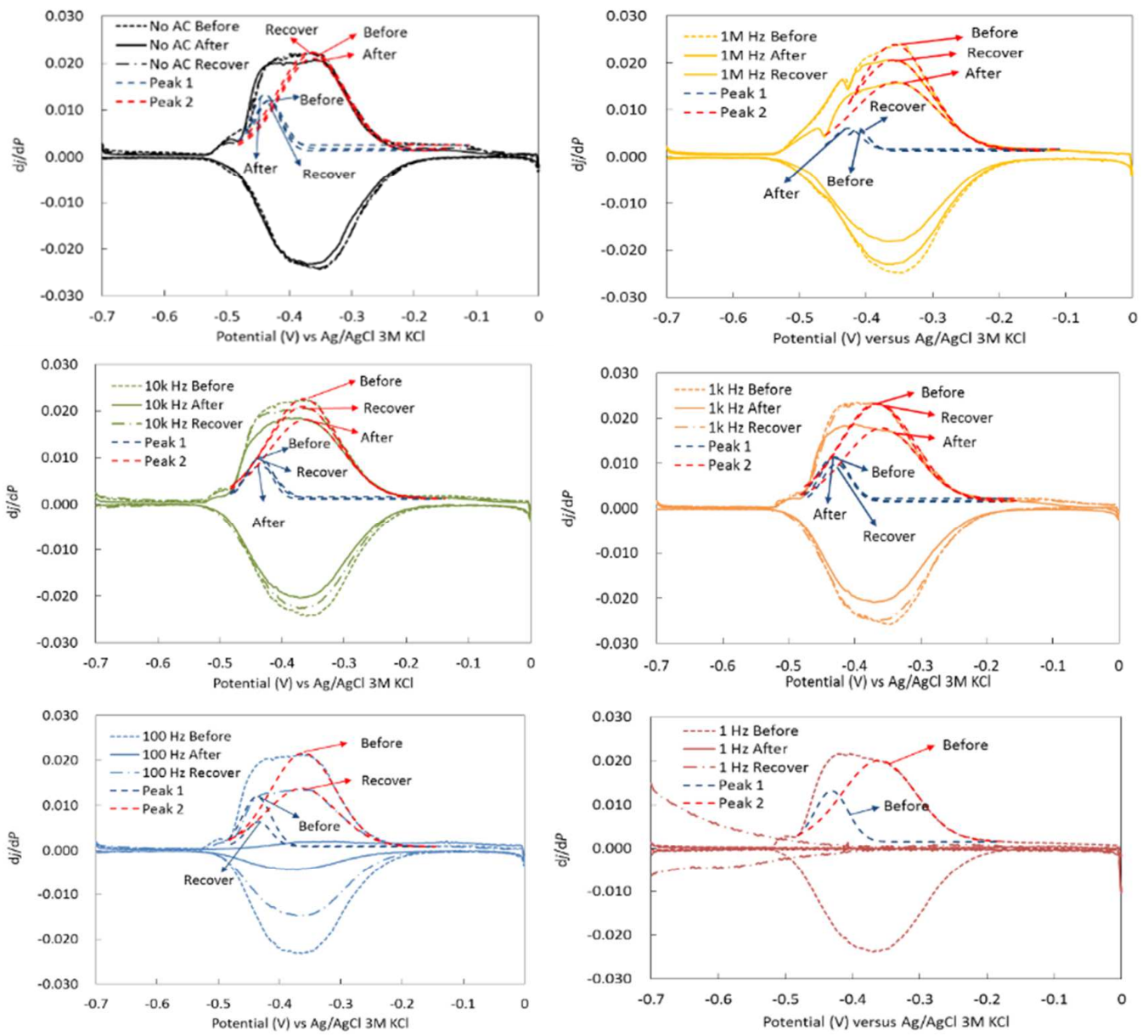

Figure S3 Turnover DCVs of anodes before, after and recovered from 1M, 10k, 1k, 100 and $1 \mathrm{~Hz}$ AC treatments and the no treatment control. 

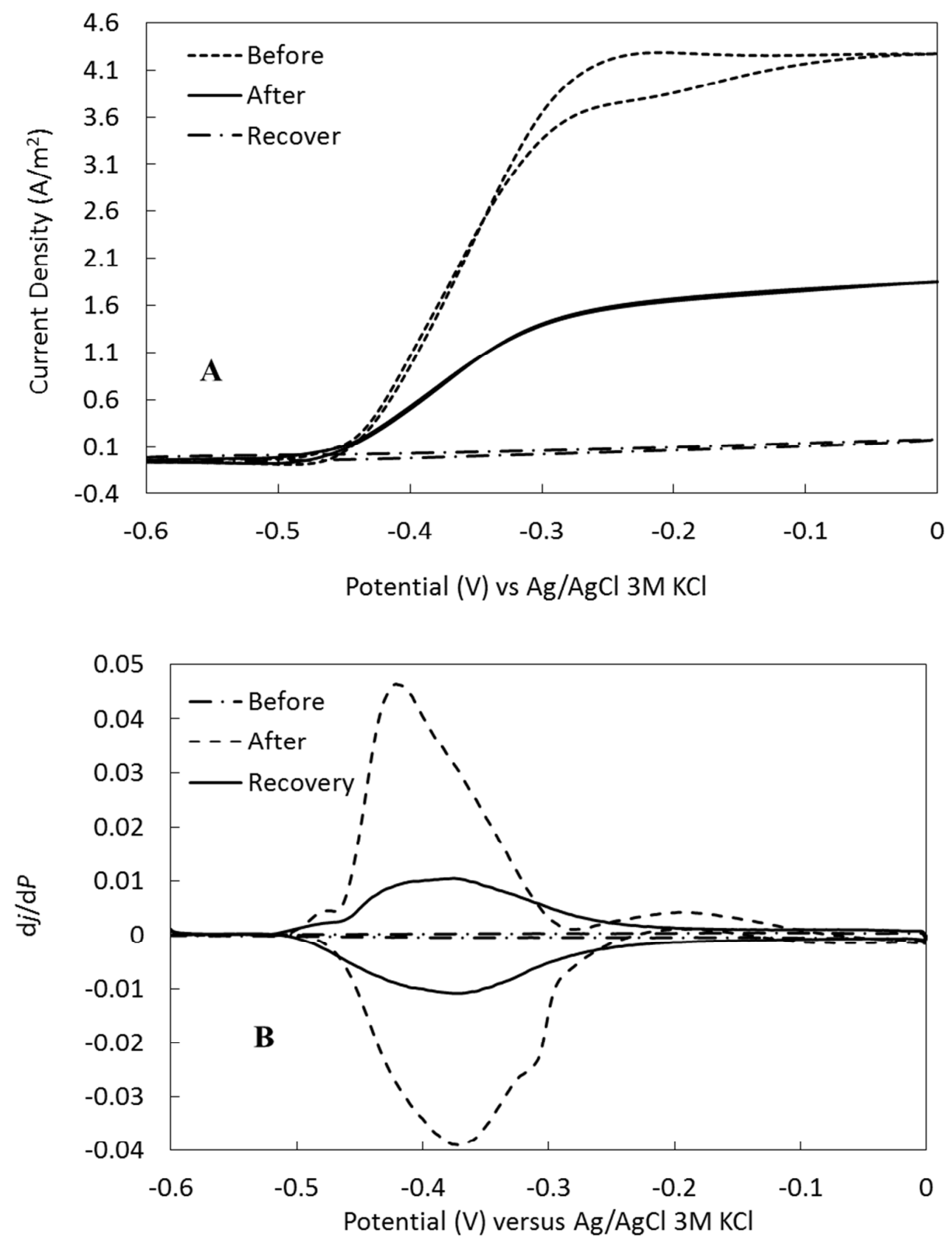

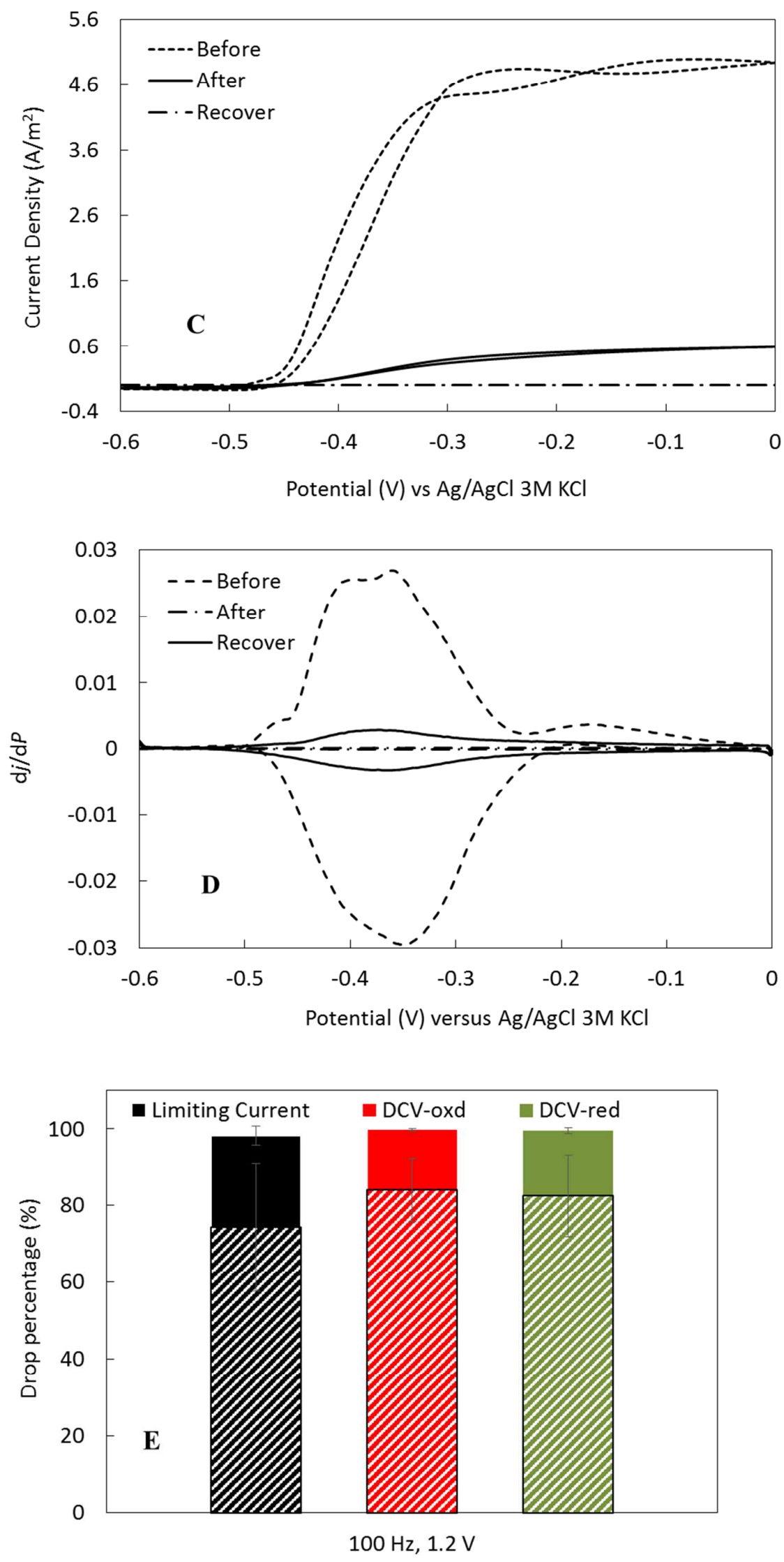
Figure S4. Turnover CVs (A, C) and DCVs (B, D) of the anodes under 1.2V, $100 \mathrm{~Hz}$ AC treatment. (before treatment, after treatment, and recovery after 12h). (E) The different percentages of biofilm responses in terms of the limiting current and DCV oxidation curve peak heights. The blank fraction (top) of each column shows the unaffected percentage of each biofilm function parameter; the solid fraction (middle) shows the recoverable percentage 12 hours after AC treatment; the shaded fraction (bottom) shows the percentage of biofilm with permanent damage.
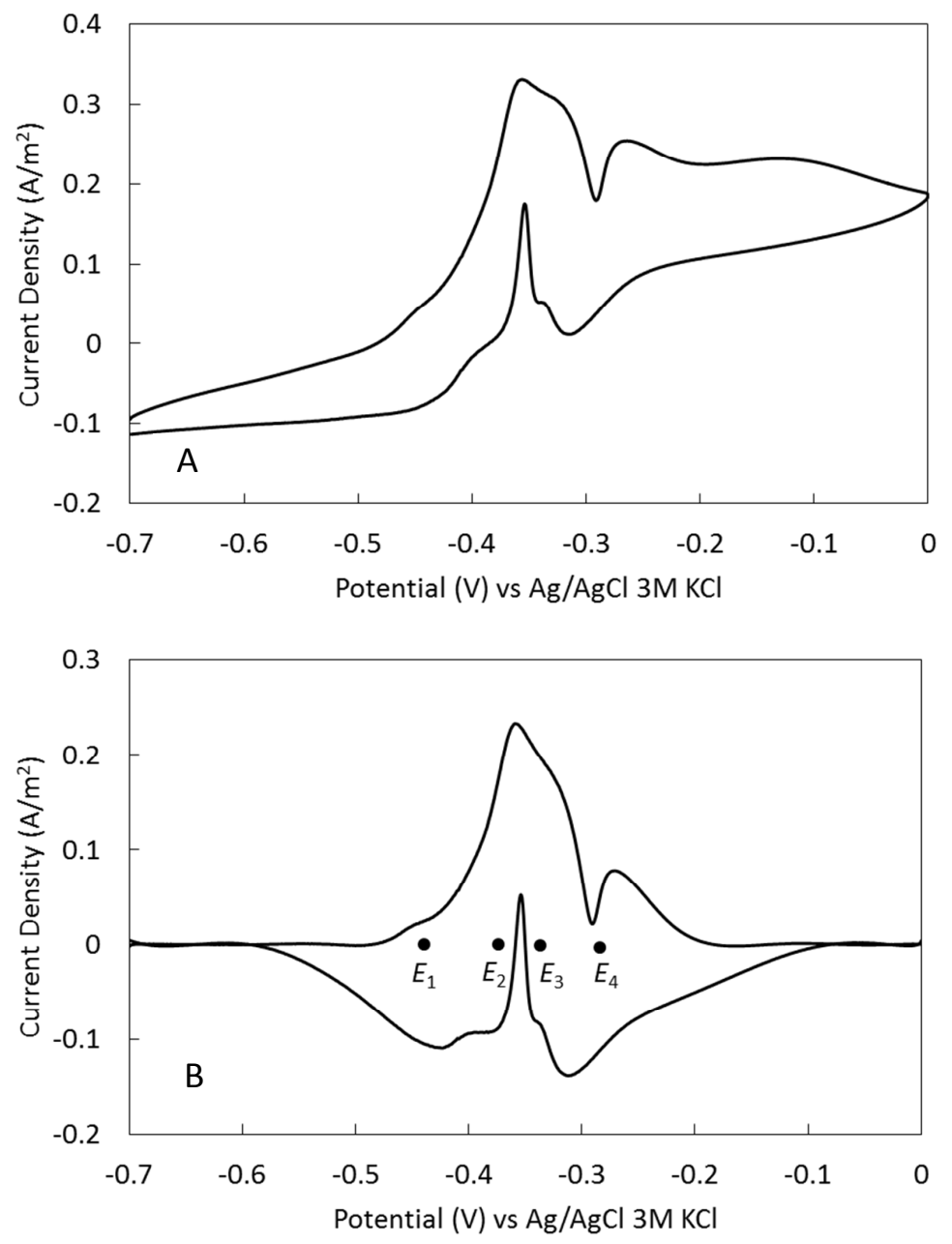

Figure S5 Typical nonturnover CV (A) and the baseline subtracted curve (B) derived from (A). 


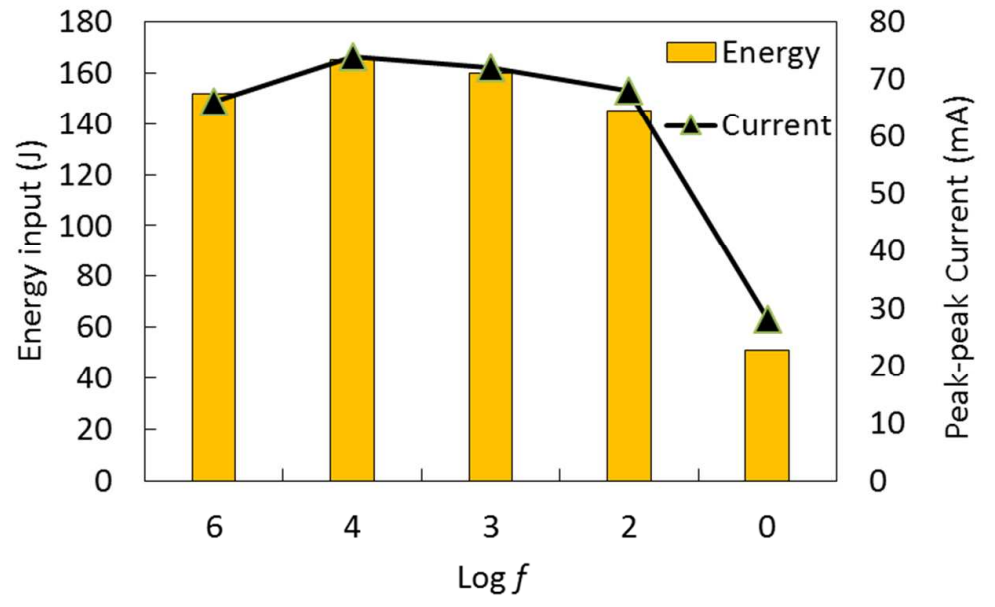

Figure S6 Peak-peak current and energy input during 30 min of AC treatments with different frequencies. $f$ is the frequency. 